\title{
The analysis of IEEE 802.11 PCF protocol based on LEO satellite Wi-Fi
}

\author{
Wen $\mathrm{He}^{*}$, Xiaofeng Tao, and Defeng Ren \\ China Academy of Space Technology (Xi'an), China
}

\begin{abstract}
In view of the problem of long propagation delay, hidden terminals and serious user conflicts in LEO satellite Wi-Fi scenario, this paper analysis the incompatibility of 802.11 DCF protocol briefly. A scheme of PCF polling mechanism based on priority is proposed and the frame format is adaptively modified. Through MATLAB simulation, the system throughput of the original scheme and the improvement scheme over the different transmission distance and nodes are analysed. The simulation results show that the improved PCF protocol can improve the throughput under the multi-user over long distances.
\end{abstract}

\section{Introduction}

More than $52 \%$ of the world's 7 billion people are unable to use the Internet normally. The main reason for this phenomenon is the lack of communication infrastructure and high network costs. The establishment of a satellite network may be able to effectively solve this predicament. In recent years, companies such as O3b and One Web are preparing to build an Internet constellation that includes hundreds of satellites. Facebook and Google also tried to achieve network coverage on the ground through solar-laser communication drones or high-altitude hot air balloons [1]. Space X plans to send 4425 satellites to the orbital plane by 2024 to provide high-speed broadband Internet services to customers around the world. Therefore, research and development on the satellite Wi-Fi system will become an upsurge. This topic focuses on analysing the MAC layer protocol of LEO satellite Wi-Fi network, laying a certain theoretical foundation for future research.

Currently, commercial Wi-Fi on the ground mostly adopts the IEEE 802.11 standard, and the media access control (MAC) layer mainly adopts Distributed Coordination Function (DCF) protocol. However, the satellite link has the characteristics of long propagation delay, hidden terminals, and serious user conflicts. Therefore, the DCF protocol shows obvious incompatibility. This paper proposes a Point Coordination Function (PCF) protocol based on the priority for long-distance multi-user wireless communication scenarios and adjusts the frame format appropriately. Finally, through MATLAB simulation, the system throughput over different transmission distances and different number of users under saturated and non-saturated conditions is analysed. Figure 1 shows the LEO satellite Wi-Fi system model used in this study.

\footnotetext{
*Corresponding author: 1162999021@qq.com
} 


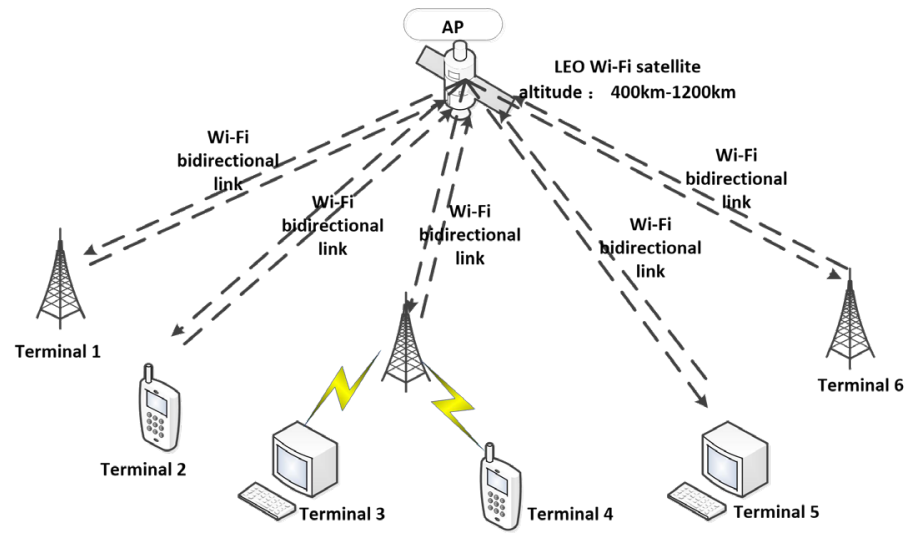

Fig. 1. LEO satellite Wi-Fi system model.

\section{A brief introduction to 802.11 PCF protocol}

The 802.11 MAC layer is divided into two modes based on competition [2]. One is a competitive DCF mode in which each node autonomously competes to acquire a channel, but its throughput performance is low [3] in the Wi-Fi scenario because of the long propagation delay, many hidden terminals, and serious user conflict, as shown in figure 2 . Another is the non-competitive PCF mode. The access point APs poll the nodes to allocate channel resources, allowing the 802.11 network to provide a more "fair" medium access mechanism.

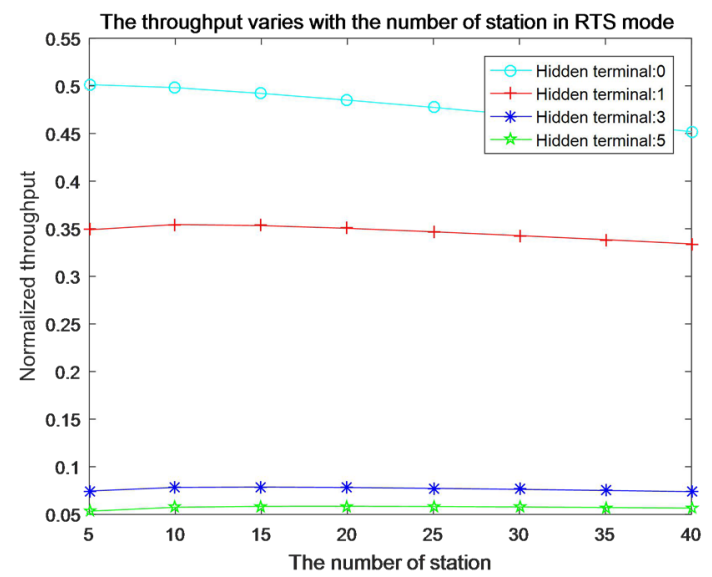

Fig. 2. The throughput of the DCF protocol varies with the number of stations at $400 \mathrm{~km}$.

The AP acts as a central coordinator (PC) and polls the nodes connected to it based on its internal polling list to see if there is data to be transmitted. During the CFP time, each node cannot actively contend for the channel due to the NAV mechanism. Therefore, unless the base station polls the node and requests its feedback data, the node cannot actively perform the transmission action.

In the PCF mode, an alternating operation mode is generally adopted. In the CFP time phase, a PCF mechanism is used. This time is protected by a virtual carrier monitoring (NAV) mechanism. The NAV is set by a Beacon frame and ended by a CF-END frame, while the $\mathrm{CP}$ stage operates according to the DCF mode. Figure 3 shows a typical PCF work flow diagram [3]. 


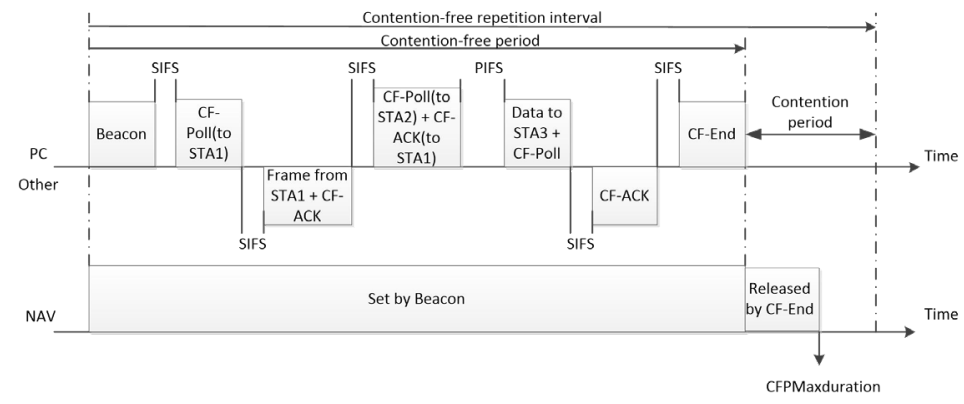

Fig. 3. PCF work flow diagram.

\section{A PCF protocol based on the priority}

In the PCF mode, the AP polls the wireless nodes according to the polling list [4]. If the nodes are not saturated, the empty polling will cause the waste of the channel, and the long distance wireless transmission of satellite-to-ground links will cause a sharp drop in throughput. Therefore, effectively reducing null frame polling is an effective means to improve the efficiency of the MAC protocol.

\subsection{The format of the frame}

Due to the incompatibility of the DCF mechanism mentioned above in the satellite Wi-Fi scenario, this paper adopts the PCF single polling method. Therefore, there is no need for Beacon and CF-END frames [5] to specify the duration of the CFP period. The transmission process of the PCF improvement mechanism requires CF-Poll, DATA, and ACK frames. The Wi-Fi MAC frame format and the frame control field format are shown in figure 4. The ACK frame format is given in figure 5.

\begin{tabular}{|c|c|c|c|c|c|c|c|c|c|c|c|c|}
\hline \multirow{2}{*}{$\begin{array}{c}2 \\
\text { Frame } \\
\text { Control }\end{array}$} & \multirow{2}{*}{\multicolumn{2}{|c|}{$\stackrel{2}{2}$}} & 6 & 6 & 6 & \multirow{2}{*}{$\begin{array}{c}2 \\
\text { Sequence } \\
\text { Control }\end{array}$} & 6 & \multicolumn{2}{|c|}{$0 \sim 2312$} & 2 & \multirow[t]{2}{*}{ Bytes } & \\
\hline & & & Address 1 & Address 2 & Address 3 & & Address & \multicolumn{2}{|c|}{$\begin{array}{l}\text { Frame } \\
\text { Body }\end{array}$} & FCS & & \\
\hline \multicolumn{2}{|c|}{ Protocol } & $\begin{array}{l}\text { Type } \\
\text { data }\end{array}$ & Sub type & To DS & $\begin{array}{c}\text { From } \\
\text { DS }\end{array}$ & $\begin{array}{l}\text { More } \\
\text { Frag }\end{array}$ & Retry & $\begin{array}{c}\text { Pwr } \\
\text { Mgmt }\end{array}$ & $\begin{array}{l}\mathrm{Mo} \\
\mathrm{Dat}\end{array}$ & & $\begin{array}{l}\text { rotected } \\
\text { Frame }\end{array}$ & Order \\
\hline Bits & 2 & & 4 & 1 & 1 & 1 & 1 & 1 & 1 & & 1 & 1 \\
\hline
\end{tabular}

Fig. 4. MAC frame format.

In the workflow of PCF mechanism [6], the destination address of DATA and CF-Poll is the same, so it can be sent as a frame at the same time. If the station transmits a simple polling frame CF-Poll, the Frame Body is 0 bytes and the frame size is 32 bytes; if the frame to be transmitted is CF-Poll + DATA, the Frame Body is a DATA byte $(0 \sim 2312)$. The frame size is (32+DATA) bytes.

\begin{tabular}{|c|c|c|c|}
\hline $\begin{array}{c}\text { Frame } \\
\text { Control }\end{array}$ & $\begin{array}{c}\text { Duration } \\
\text { /ID }\end{array}$ & $\begin{array}{c}\text { Receiver } \\
\text { Address }\end{array}$ & FCS \\
\cline { 1 - 2 } &
\end{tabular}

Fig. 5. ACK frame format.

Since the ACK (figure 5) is a response frame that successfully receives the previous frame of data and is different from the destination address of other frames, it cannot be transmitted as the same frame, but can be transmitted by the station at the same time. If the data frame is ACK, the frame size is (14+Phy_hdr) bytes; if it is CF-Poll + ACK, the frame size is $(32+14+$ Phy_hdr) bytes; if it is CF-Poll + DATA + ACK, the frame size is $(32+$ DATA+14+Phy_hdr) bytes. Phy_hdr is a physical header of 24 bytes. 
The 802.11 frame has a maximum of 4 address fields. In this study, Address 1 represents the address of the STA to be polled, Address 2 represents the BSSID address, which is the address of the AP, Address 3 represents the destination address of the acknowledgment frame ACK, and Address 4 represents the destination address of the data frame to be transmitted.

\subsection{Algorithm flow}

LEO satellite link distance is at least $400 \mathrm{~km}$ and propagation delay is up to $1.33 \mathrm{~ms}$. According to the requirements of the PCF protocol [7], the satellite access point AP polls the ground terminal stations in sequence according to the polling table. If there is no data transmission at the station, the AP waits for PIFS (PIFS=2*Propagation Time + SIFS), and then polls the next station. Therefore, empty frame polling will waste channel resources and reduce MAC layer protocol efficiency. To improve the throughput of the satellite Wi-Fi system, it is necessary to poll the empty frames as little as possible. Therefore, a PCF polling based on priority mechanism is proposed. The access point dynamically changes its polling priority based on whether or not the station has data transmitted. The higher the priority, the more polling times.

Assuming that the system has $\mathrm{M}$ stations, the maximum priority is $\mathrm{M}$. At the first poll, each station has a priority of 1 . The workflow of the PCF is as the following: The access point AP polls the station in the polling table in sequence. If the AP has data to be sent, the channel transmits the data frame CF-Poll + DATA. After the station receives the CF-Poll, if the buffer has data to be transmitted, the data frame DATA is sent to the access point after the SIFS duration, and when the station priority is not 1 , the priority is decremented by 1 ; otherwise, the station does not change the priority. If the polling station has no data to be transmitted, the AP waits for the PIFS duration and polls the next station. When the station priority is not $\mathrm{M}$, the priority of the station is incremented by 1; otherwise, the station does not change. In addition, when the station successfully receives the data frame sent by the $\mathrm{AP}$, the response frame ACK is also returned at the same time. For the AP, when it successfully receives the DATA frame, and the AP has data to be transmitted, this polling transmits the data frame CF-Poll + DATA + ACK. The destination address of CF-Poll and DATA is the station to be polled, and the destination address of the ACK is the last polling station. Figure 6 shows the polling operation of a polling list during the PCF protocol. After each round of polling, the number of stations with the highest priority in the polling table is removed, and a new round of polling is performed until the maximum number of cycles (determined by the number of priority classes). After that, the polling table is updated according to the station priority, and the next polling round will start. Besides, When all stations have a priority of $\mathrm{M}$, the initialization priority is 1 .

\begin{tabular}{|c|c|c|c|c|c|c|}
\hline \multicolumn{6}{|c|}{$\begin{array}{l}\mathrm{P}=\text { The number of } \\
\text { priority classes }=4\end{array}$} & \multirow{2}{*}{$\begin{array}{c}\mathrm{R}=\text { Number of } \\
\text { nodes polled } \\
\text { per round } \\
\mathrm{R}=5\end{array}$} \\
\hline $\begin{array}{c}\text { Polling } \\
\text { period is } 1\end{array}$ & 1 & 2 & 3 & 3 & 4 & \\
\hline $\begin{array}{c}\text { Polling } \\
\text { period is } 2\end{array}$ & 1 & 2 & 3 & 3 & & $R=4$ \\
\hline $\begin{array}{c}\text { Polling } \\
\text { period is } 3\end{array}$ & 1 & 2 & & & & $R=2$ \\
\hline $\begin{array}{c}\text { Polling } \\
\text { period is } 4\end{array}$ & $1 \leftarrow$ & & & & & $\mathrm{R}=1$ \\
\hline
\end{tabular}

Fig. 6. Polling operation.

The specific algorithm is shown in figure 7. 


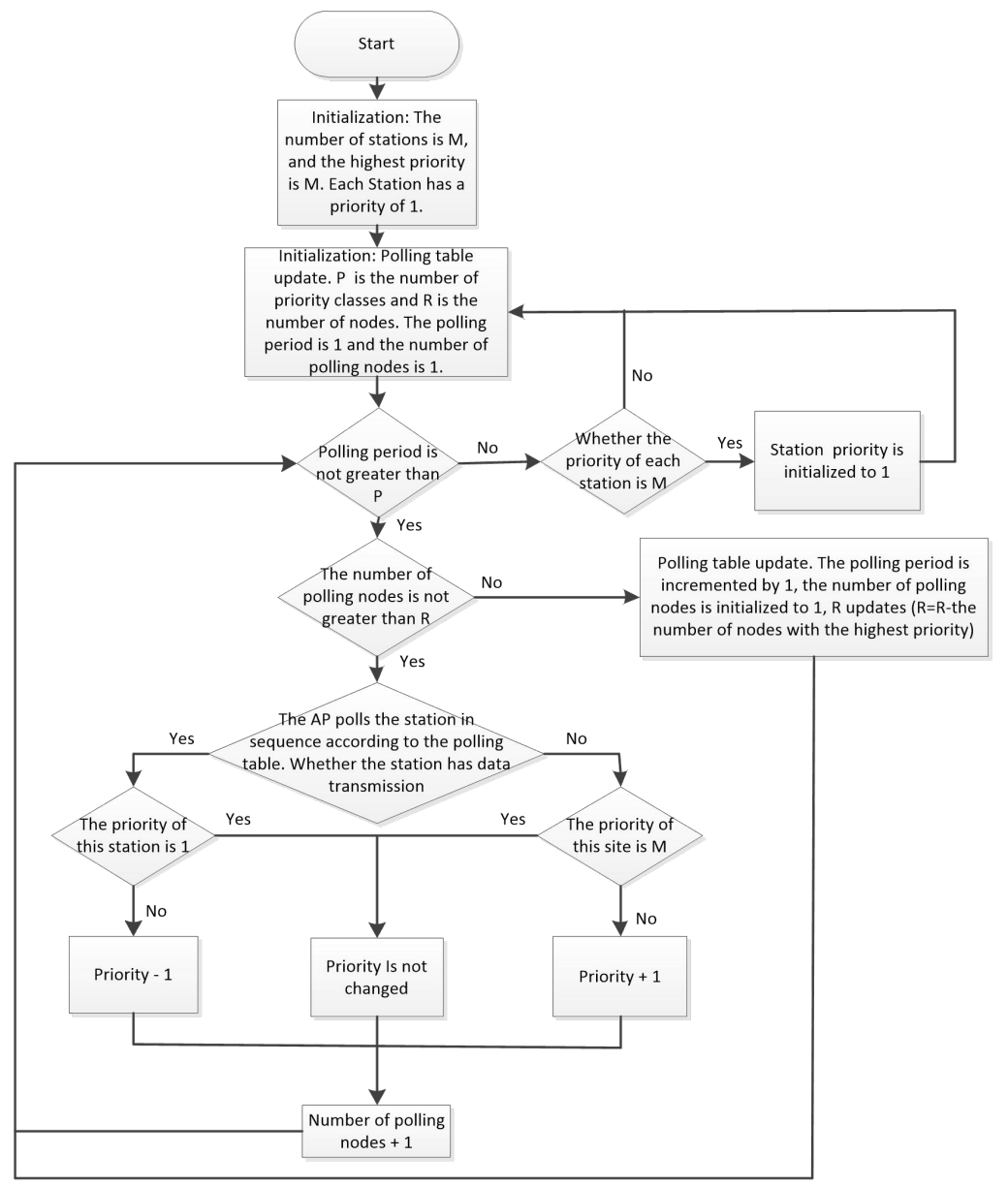

Fig. 7. Polling algorithm flow chart.

The PCF polling algorithm based on the priority adjusts the priority dynamically according to the traffic volume of the station, ensuring frequent polling of stations with large traffic volume, reducing the polling waste of empty frames and improving the system throughput.

\section{The simulation verification}

Based on the above improved algorithm, the performance of the PCF mechanism is simulated and analysed through MATLAB. The following shows the variation of the normalized throughput of the system with the transmission distance and the number of nodes. Where,

$$
\text { Throughput }=\frac{\text { The amount of data successfully transferred in unit time }}{\text { The length of the unit time }}
$$

$$
\text { Normalized throughput }=\frac{\text { Throughput }}{\text { Channel transmission rate }}
$$

(1) In the saturation state, the simulation of the original PCF polling mechanism where the priority is not set. 


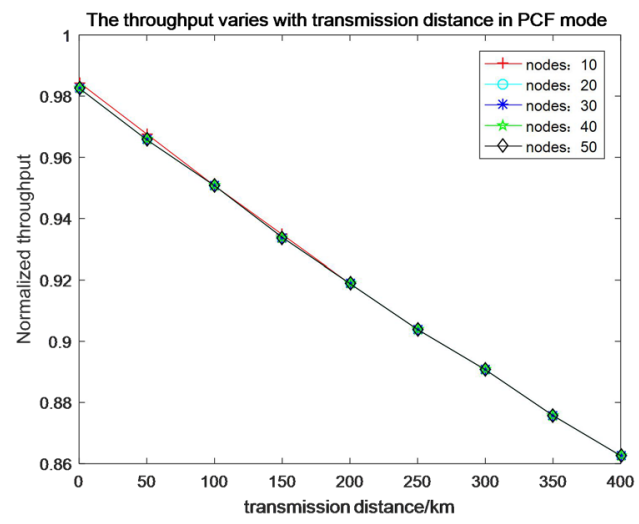

Fig. 8. Throughput versus transmission distance: PCF protocol in the saturation state.

We can see from the figure 8 that the system throughput decreases as the transmission distance increases. Since data is transmitted at any time, the throughput does not depend on the number of users. However, the increase of the transmission distance will waste the channel resources in the information transmission, which will degrade the system performance. When the low-orbit satellite height is $400 \mathrm{~km}$, the normalized throughput can reach $86 \%$.

(2) In the unsaturated state, the simulation of the original PCF polling mechanism where the priority is not set.

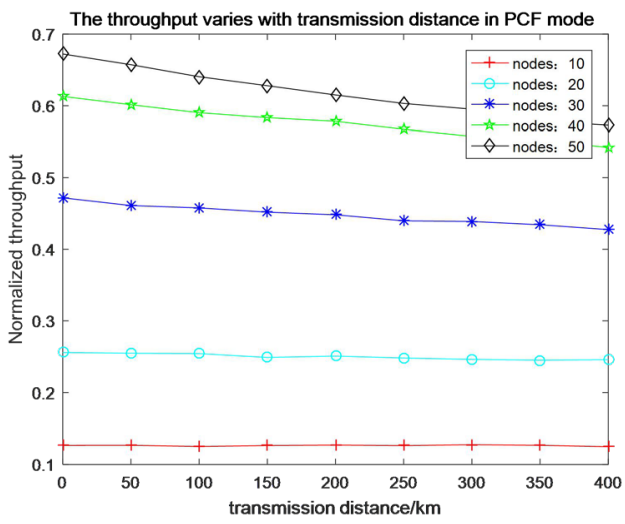

Fig. 9. Throughput versus transmission distance: PCF protocol in the unsaturation state.

Due to the presence of empty frame polling, the high throughput effect of the saturation state cannot be achieved. The increase of users makes the system traffic increase, so the probability of empty frame polling will decrease and the throughput will increase. The more users there are, the more obvious the throughput decreases as the distance increases. When the number of users is small, the increase of the distance makes the buffer data of each node pile up, the probability of empty frame polling decreases, and the throughput increases. However, the increase of the distance causes the waste of channel resources and the throughput decreases. Thus, the throughput is insensitive to changes in the distance of the small-user system as is shown in figure 9. When the low-orbit satellite height is $400 \mathrm{~km}$, the normalized throughput of different users can reach $13 \%-57 \%$.

(3) In the unsaturated state, the simulation of the PCF polling mechanism based on the priority. 


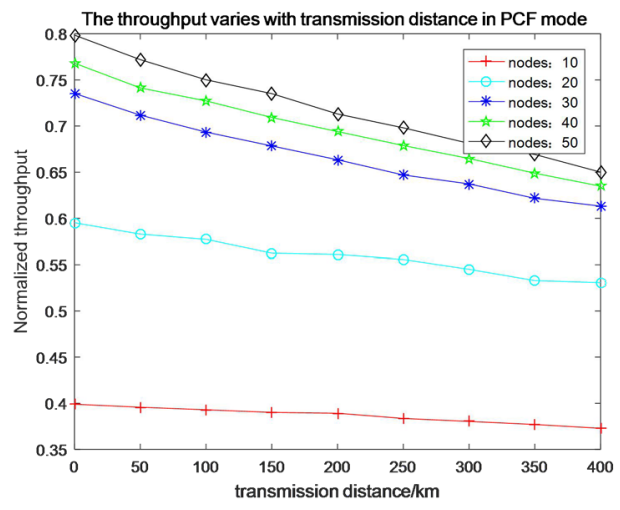

Fig. 10. Throughput versus transmission distance: PCF protocol based on priority.

The simulation results of the PCF polling mechanism based on the priority are similar to the above. When the low-orbit satellite height is $400 \mathrm{~km}$, the normalized throughput of different users can reach $38 \%-65 \%$. Compared with figures 9 and 10 , we can see that the modified algorithm can improve the system throughput.

(4) In order to compare the superiority of the PCF polling mechanism in which the priority changes with the number of nodes in (3), the simulation of PCF polling algorithm with the maximum priority of 5 and 10 in the unsaturated state is given in figure 11 and 12 .

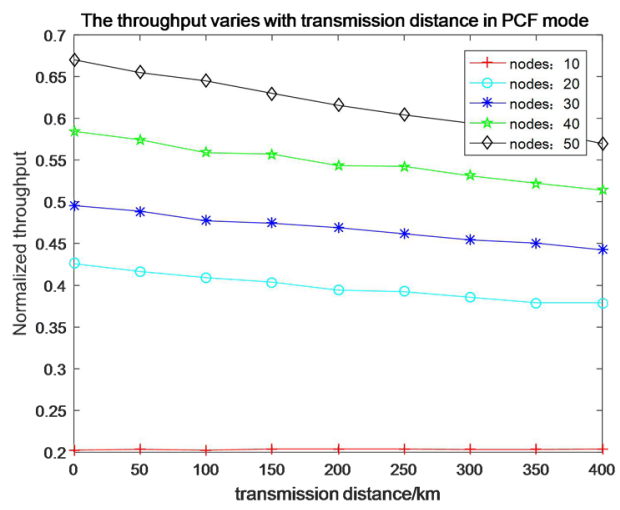

Fig. 11. Throughput versus transmission distance: the maximum priority is 5 .

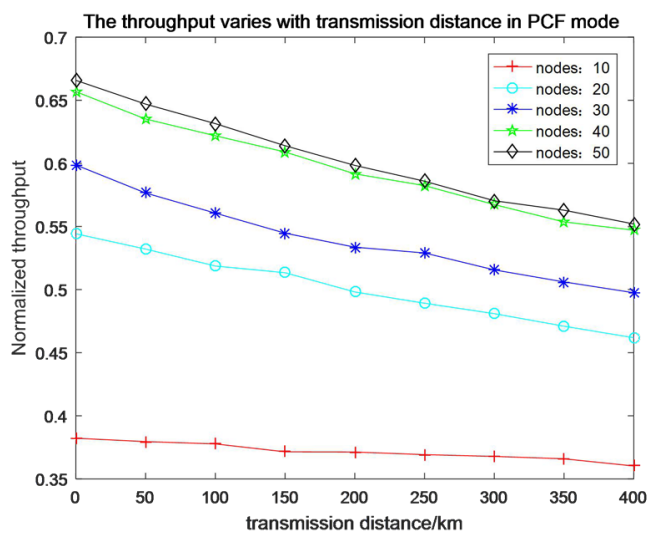

Fig. 12. Throughput versus transmission distance: the maximum priority is 10 . 
With a transmission distance of $400 \mathrm{~km}$, the normalized throughput for different users with a maximum priority of 5 and 10 is $20 \%-57 \%$ and $36 \%-55 \%$, respectively. Compared with the result in figure 10 , the effect of the algorithm with a fixed maximum priority is slightly worse.

Through the above simulation results, it can be seen that multi-user wireless communications in a long distance environment, the effect of the PCF protocol is much better than the DCF protocol affected by the propagation delay and the hidden terminal. Under the unsaturated condition, the improved scheme based on the priority PCF polling mechanism improves the system throughput, and the priority number changes dynamically with the number of nodes.

\section{Conclusions}

This article first briefly introduced the relevant knowledge of 802.11 PCF protocol. In view of the characteristics of long propagation, hidden terminals and serious user conflicts in the satellite Wi-Fi, we made appropriate improvements to the PCF protocol, and proposed a $\mathrm{PCF}$ polling algorithm based on the priority. The improved protocol changes the traditional access mechanism of PCF-DCF to a single PCF polling mechanism and modifies the MAC layer frame format. Finally, the MATLAB simulation verifies that under saturated conditions, the throughput only decreases with the distance. Under unsaturated conditions, the more users, the more the throughput is affected by distance.

\section{References}

1. Q.Lu, Z.C.Zhou, F.Li, WIFI Application Based on Satellite Communications [J]. International Space, 2015(11): 34-39.

2. S.MATTBEW GAST, 802.11 Wireless Network Authoritative Guide [M]. Second Edition. Nanjing: Southeast University Press, (2007)

3. N.Wang, Experimental Study of 2P MAC Protocol in Long Distance Wireless Mesh Networks [D].Tianjin University, (2012)

4. Z.Guan, Z.J.Yang, M.He, et al. Energy-efficient analysis of an IEEE 802.11 PCF MAC protocol based on WLAN [J]. Journal of Ambient Intelligence \& Humanized Computing, 2018 (2):1-11.

5. B.Sikdar. An Analytic Model for the Delay in IEEE 802.11 PCF MAC-Based Wireless Networks [J]. Wireless Communications IEEE Transactions on, 2007, 6 (4):1542-1550.

6. R Palacios, G Mengistie Mekonnen, J Alonso-Zarate, et al. Analysis of an energyefficient MAC protocol based on polling for IEEE 802.11 WLANs[C]// IEEE International Conference on Communications. IEEE, 2015:5941-5947.

7. L.Feng, J.Li, X.Lin. A New Delay Analysis for IEEE 802.11 PCF [J]. IEEE Transactions on Vehicular Technology, 2013, 62 (8):4064-4069. 